\title{
Balmer lines emission region in NGC 3516: Kinematical and physical properties
}

\author{
L. Č. Popović ${ }^{1}$, E. G. Mediavilla ${ }^{2}$, A. Kubičela ${ }^{1}$, and P. Jovanović ${ }^{1}$ \\ 1 Astronomical Observatory and Isaac Newton Institute of Chile, Branch in Yugoslavia, Volgina 7, 11160 Belgrade 74, Serbia, \\ Yugoslavia \\ 2 Instituto de Astrofsica de Canarias C/ Va Làctea, s/n 38200 - La Laguna (Tenerife), Spain
}

Received 14 January 2002 / Accepted 15 May 2002

\begin{abstract}
We have studied the Balmer line shapes of NGC 3516 in order to find the structure of central Emission Line Region (ELR) and the physical parameters of the emitting plasma. The shapes of these broad emission lines show evidences of a multicomponent origin and also features which could be identified as the peaks of a rotating disc. We have proposed a two component Broad Line Region (BLR) model consisting of an inner Keplerian relativistic disc and an outer structure surrounding the disc which is composed by Broad Line and Narrow Line Regions (BLR and NLR). Adopting a mass of $\sim 4 \times 10^{7} M_{\odot}$ for the central object we found that the emitting disc is located from $0.004 \mathrm{pc}$ to $0.018 \mathrm{pc}$. Using a well known method for laboratory plasma diagnostic, the Boltzmann plot, we estimated the electron temperature $(\approx 12500 \mathrm{~K})$ and electron density $\left(\sim 10^{15} \mathrm{~cm}^{-3}\right)$ of the disc and the surrounding region $\left(T_{\mathrm{e}} \approx 20000 \mathrm{~K}\right.$ and $\left.N_{\mathrm{e}} \sim 10^{15} \mathrm{~cm}^{-3}\right)$.
\end{abstract}

Key words. galaxies: individual: NGC 3516 - galaxies: Seyfert - line: profiles - accretion, accretion disks

\section{Introduction}

One of the most attractive ideas about the nature of the Broad Line Region (BLR) in Active Galactic Nuclei (AGN) is the existence of a disc generating a significant part of the Broad Emission Lines (BEL). Although some features which could be associated to BLR discs were previously detected (see e.g. Perez et al. 1988), the discovery of the double-peaked Balmer Lines in Arp 102B by Chen et al. (1989), gave a fundamental support to the disc geometry for the BLR. The line profiles of this object could be well fitted by the predictions of a model for line emission from a relativistic Keplerian disc (Chen $\&$ Halpern 1989). In spite of the success of the disc model in Arp 102, the fraction of AGNs with double-peaked emission lines (see e.g. Chen et al. 1989; Chen \& Halpern 1989; Eracleous \& Halpern 1994) is small, and the observational support to the existence of discs is not statistically significant (Eracleous \& Halpern 1994; Eracleous 1999). The doublepeaked line profile, characteristic of a Keplerian relativistic disc has been mainly observed in radio-loud AGNs (Eraclous \& Halpern 1994; Eraclous 1999). However, disc profiles have been also recently detected in two lower activity galaxies, such as NGC 1097 and NGC 3065 that present a characteristic LowIonization Nuclear Emission-Line Regions (LINERs) spectrum (Storchi-Bergmann et al. 1997; Eraclous \& Halpern 2001 and references therein).

Send offprint requests to: L. Č. Popović, e-mail: lpopovic@aob.bg.ac.yu
However, the presence of two-peaked lines should not be required as a necessary condition to the existence of a disc geometry (see e.g. van Groningen 1987; Sulentic et al. 1998; Sulentic 1999; Popović et al. 2001, 2002). One possibility is that the central dip of the line associated to the disk geometry may be masked by broad emission coming from elsewhere. A two component model including an accretion disk and a region with different geometry covering the disk has satisfactorily described the emission lines of Akn 120 (Popović et al. 2001) and III Zw 2 (Popović et al. 2002).

The existence of an accretion disc is also supported in some AGN by the shape of the $\mathrm{Fe} \mathrm{K}_{\alpha}$ line (Nandra et al. 1997). This is the case of NGC 3516 where the $\mathrm{Fe}_{\alpha}$ line has been modeled according to a disc geometry. Nandra et al. $(1997,1999)$ fitted an integrated $\mathrm{Fe} \mathrm{K}$ profile deriving the disc inclination for the Schwarchild metric $i=35_{-2}^{\circ+1}$ and for the Kerr metric $i=0_{-0}^{\circ+19}$. More recently, Pariev et al. (2001) have also studied the $\mathrm{Fe}_{\alpha}$ emission obtaining a disc inclination of about $27^{\circ}$. The $\mathrm{H} \alpha$ line was fitted in an earlier work by Sulentic et al. (1998) with a model including a disc of inclination $i \approx 20^{\circ}$ and $R_{\text {out }} \approx 1.5 \times 10^{5} R_{\mathrm{g}}\left(R_{\mathrm{g}}=G M / c^{2}\right)$. Although, the Full With at Half Maximum $(F W H M)$ of $\mathrm{H}_{\alpha}$ is not as large as in typical disc-like emitters (that are twice wider than in ordinary AGNs, see Eracleous \& Halpern 1994) as well as the narrow line ratios are not comparable with ones in LINERs, the blue shoulder in Balmer lines indicates the presence of disc-like emission.

In this paper we will present an analysis of the shapes of the Balmer lines of NGC 3516 based in HST observations of this 
object (one of the best investigated Sy 1 galaxies in all wavelength ranges, see e.g. Golev et al. 1995; Nandra et al. 1997, 1999; Goad et al. 1999; Edelson et al. 2000; Chiang \& Blaes 2001; Reeves et al. 2001). The aims of this work are: (i) to try to fit the Balmer lines of NGC 3516 with a two component model including emission from a disc (or disc-like region) and a structure surrounding the disc; (ii) to compare the results of this study with the ones obtained from the $\mathrm{Fe} \mathrm{K}_{\alpha}$ line (Nandra 1997, 1999; Pariev 2001); and (iii) to discuss the physical conditions in the region emitting the broad Balmer lines.

\section{Observations}

We use HST observations obtained with the Space Telescope Imaging Spectrograph (STIS), covering the wavelength ranges

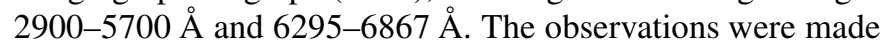
on April 13-16, 1998 (wavelength range 2900-5600 $\AA$, in the frame of the campaign monitoring of NGC 3516 with HST, RXTE and ASCA, for more details see Edelson et al. 2000) and on January 18, 2000 (wavelength ranges 2900-5600 ̊ and 6295-6867 $\AA$ ). Two different gratings, G430L and G750M, were used to cover all the observed spectral range. The dispersion of the spectra were of $0.556 \AA /$ pixel and $2.747 \AA /$ pixel in the wavelength ranges $6295-6867 \AA$ and $2900-5600 \AA$, respectively. The spectra were reduced by the HST team. We transform to zero redshift the wavelength scale taking into account the cosmological red-shift $(z=0.00884$, Véron-Cetty $\&$ Véron 2000). After that we estimate and subtract the continuum defined taken as reference the wavelengths: $3750 \AA$, $3900 \AA$, $4050 \AA, 4200 \AA, 4450 \AA, 5100 \AA, 5600 \AA, 6400 \AA$ and $6750 \AA$. Finally, as the variation in the continuum is very small (about 3\%, see e.g. Edelson et al. 2000; Chiang \& Blaes 2001) and the changes in the Balmer lines shape were not noticeable, all the available spectra from the 1998 and 2000 observations were respectively averaged.

\section{Results}

\subsection{Gaussian analysis of the Balmer line profiles}

A multi-Gaussian analysis is used to do a first study of the Balmer lines. We fit each line with a sum of Gaussian components using a $\chi^{2}$ minimalization routine. In Figs. $1-5$ we can see clear evidences of substructure in the broad emission component. In all the line profiles we can recognize a significant blue shoulder that can be interpreted as an evidence of the blue peak of a disc-like emission. Some evidences of a structure can be also seen in the red wing, specially in the $\mathrm{H} \beta$ and $\mathrm{H} \delta$ lines where the change in slope is more noticeable. We use two Gaussians to reproduce these blue and red features and another one to account for the core broad emission. We suppose that the narrow components and the satellite lines can also be represented by one or more Gaussian components. In the next sections we describe the restrictions imposed on these lines.

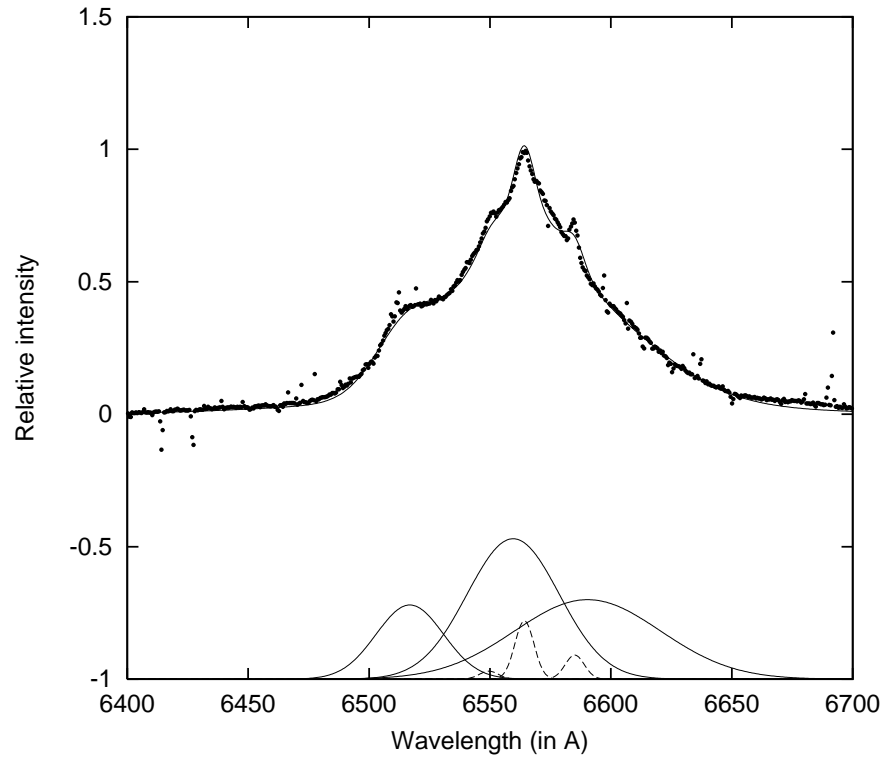

Fig. 1. Decomposition of $\mathrm{H} \alpha$ line. The dots represent the observation and solid line is the best fit. The Gaussian components are shown at the bottom. The dashed line at the bottom represent satellite $\mathrm{N}$ II lines and a narrow $\mathrm{H} \alpha$ component.

\subsection{1. $\mathrm{H} \alpha$}

We assume that the $[\mathrm{NII}] \lambda \lambda 6548,6583$ and the $\mathrm{H} \alpha$ narrow components have the same redshift and full widths proportional to their wavelengths (broken lines at the bottom of Fig. 1). Taking into account that the two [NII] lines belong to the transition between the same multiplet we assume that their intensity ratio is 1:2.96 (see e.g. Wiese et al. 1966).

\subsection{2. $\mathrm{H} \beta$}

To limit the number of free parameters in the fit of the $\mathrm{H} \beta$ line we have also set some a priori restrictions on the narrow components and satellite lines (Popović \& Mediavilla 1997; Popović et al. 2001, 2002). In the first place, the three narrow Gaussians representing the two [OIII] $\lambda \lambda 4959,5007$ lines and the narrow $\mathrm{H} \beta$ component are preconditioned to have the same redshift and full widths proportional to their wavelengths. In the second place, we have linked the intensity ratio of the two [OIII] lines according to the atomic value (line strength), $1: 3.03$. Finally, we have included in the fit a shelf of Fe II template (Korista 1992; Popović et al. 2001, 2002).

\subsection{Results from the Gaussian analysis}

The results of the Gaussian decomposition are represented in Figs. 1-6 and in the Table 1. As one can see from Figs. 1-5 the Balmer lines can be very well fitted with three broad Gaussian components; red- and blue-shifted and a central one. The ratio of intensities of the red- and the blue-shifted as well as the central Gaussian components is different for different lines, but in all cases the blue component is narrower than the red one.

In Table 1 the parameters of Gaussian components of the Balmer lines are presented; $z$ is the shift of the components with 
Table 1. The parameters of the Gaussian components ( $W$ and $z$ ) of the Balmer lines. $<$ Bal $\rangle_{\mathrm{av}}$ represents the averaged value of the parameters. $\mathrm{rms}=\sqrt{\sum_{i}^{n}\left(x_{i}-<x>\right)^{2} / n}$. $W$ is given in $\mathrm{km} \mathrm{s}^{-1}$.

\begin{tabular}{ccccccccr}
\hline \hline Line & $W_{\mathrm{B}}$ & $z_{\mathrm{B}}$ & $W_{\mathrm{C}}$ & $z_{\mathrm{C}}$ & $W_{\mathrm{R}}$ & $z_{\mathrm{R}}$ & $W_{\mathrm{NLR}}$ & $z_{\mathrm{NLR}}$ \\
\hline $\mathrm{H}_{\alpha}$ & 840 & -0.0070 & 1220 & -0.0005 & 1270 & 0.0042 & 260 & 0.00023 \\
$\mathrm{H}_{\beta}$ & 1500 & -0.0083 & 1680 & -0.0008 & 2550 & 0.0057 & 390 & -0.00026 \\
$\mathrm{H}_{\gamma}$ & 1200 & -0.0080 & 1590 & 0.0 & 2400 & 0.0038 & 420 & -0.00020 \\
$\mathrm{H}_{\delta}$ & 1140 & -0.0075 & 1500 & -0.0008 & 1950 & 0.0043 & 480 & -0.00025 \\
$\mathrm{H}_{\varepsilon}$ & 750 & -0.0070 & 1080 & -0.0010 & 2400 & 0.0043 & 480 & -0.00025 \\
\hline$<$ Bal $>_{\mathrm{av}}$ & 1086 & -0.00756 & 1414 & -0.00062 & 2114 & 0.00446 & 406 & -0.00015 \\
$\mathrm{rms}$ & \pm 300 & \pm 0.00058 & \pm 254 & \pm 0.00039 & \pm 523 & \pm 0.00072 & \pm 90 & \pm 0.00021 \\
\hline
\end{tabular}

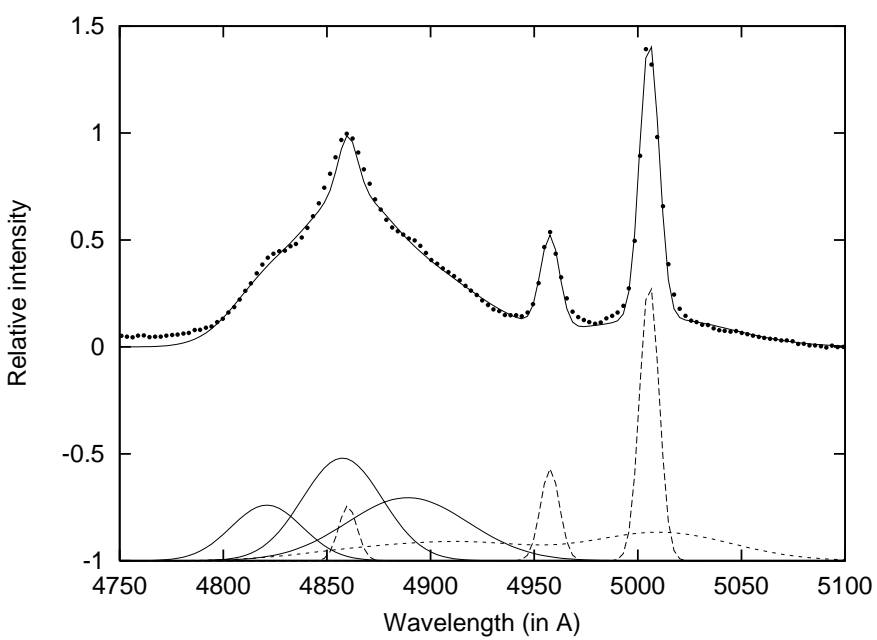

Fig. 2. The same as in Fig. 1, but for $\mathrm{H}_{\beta}$. The dashed lines at bottom represent the Fe II template and [OIII] narrow lines.

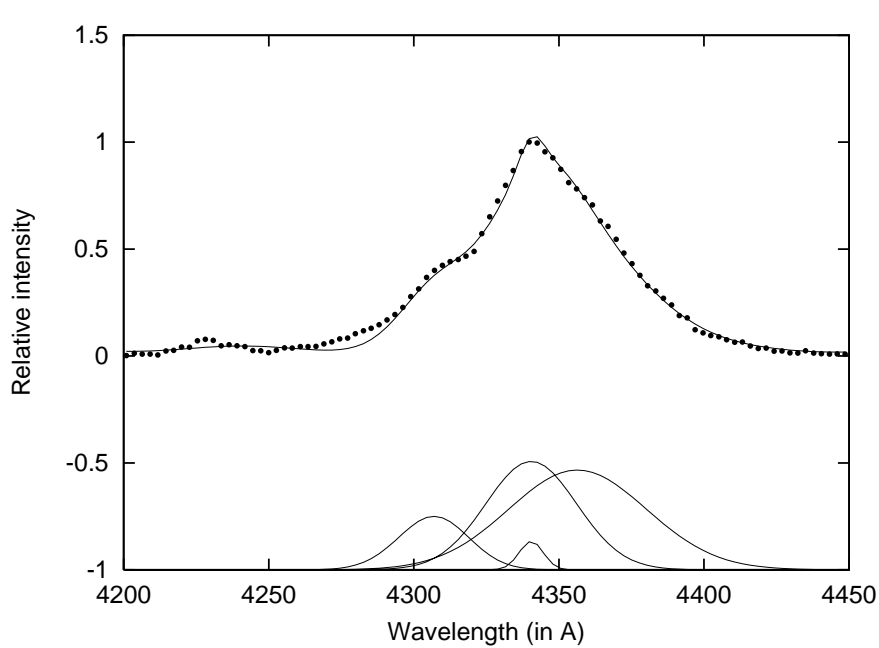

Fig. 3. The same as in Fig. 1, but for $\mathrm{H}_{\gamma}$.

respect to the rest transition wavelength $\left(z=\Delta \lambda / \lambda_{0}\right)$ and $W$ is the width of the Gaussian profile which is connected with Full Width at Half Maximum $(F W H M)$ as $F W H M=2 W \sqrt{\ln 2}$. We have included in Table 1 the widths of all the NELs, but only the width corresponding to the narrow $\mathrm{H} \alpha$ can be considered as well resolved and represents the dynamics of the emitters in NLR. The spectral resolution of the G430L grating is not good enough to resolve the widths of the other Balmer NELs.

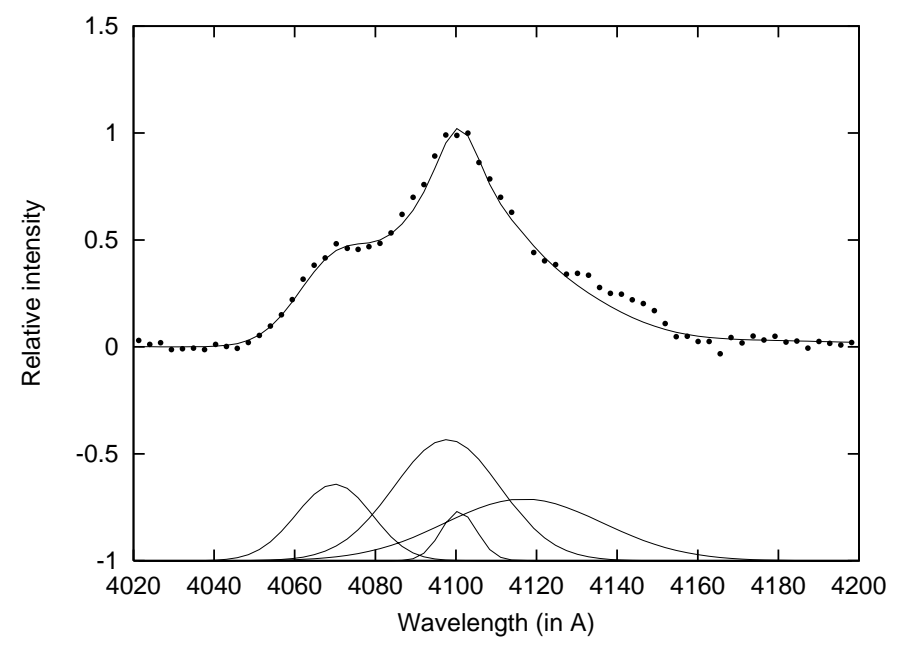

Fig. 4. The same as in Fig. 1, but for $\mathrm{H}_{\delta}$.

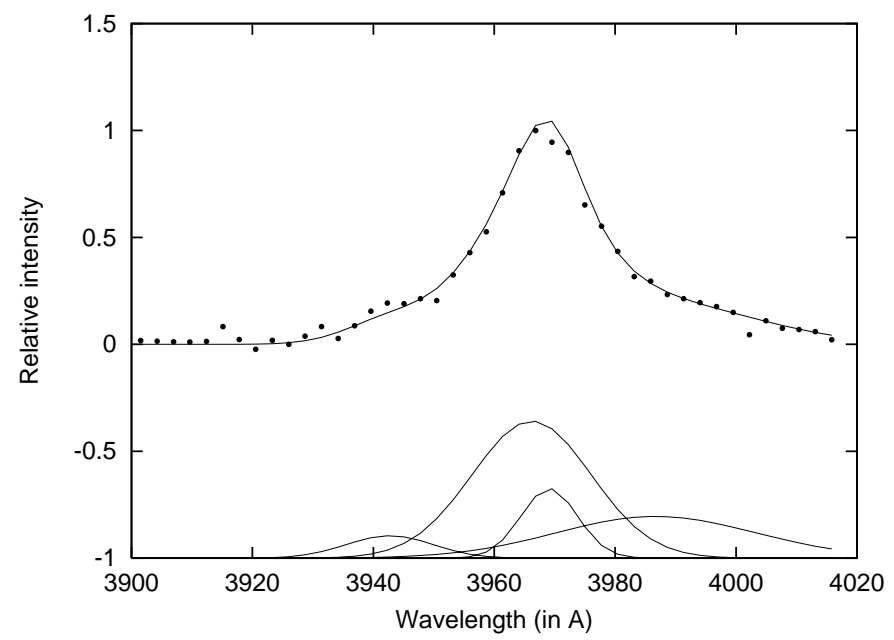

Fig. 5. The same as in Fig. 1, but for $\mathrm{H}_{\varepsilon}$.

In Fig. 6 we have represented the FWHM (width) of the different broad Gaussian components versus their centroid velocities (relative to the systemic one). The different components appear well separated in this diagram, showing the consistence of the multi-Gaussian decomposition and giving support to the physical relevance of the features represented by them. By inspection of Figs. 1-6 and Table 1 we can derive two main 


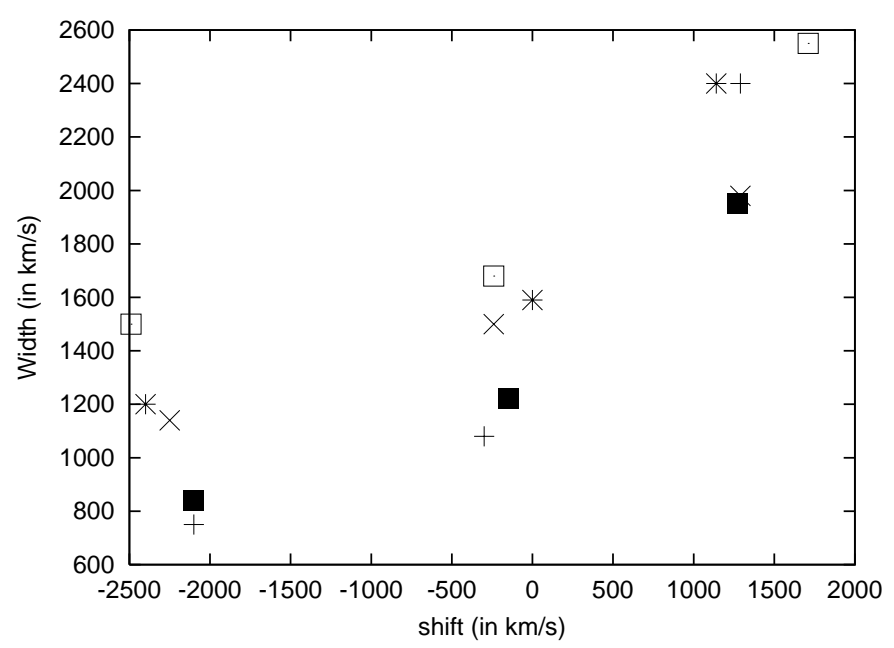

Fig. 6. The $F W H M$ of the broad Gaussian components as a function of their systemic shifts for Balmer lines of NGC 3516. The used notation is: $(\mathrm{X})$ for $\mathrm{H} \varepsilon,(+)$ for $\mathrm{H} \delta,(*)$ for $\mathrm{H} \gamma$, open squares for $\mathrm{H} \beta$ and full squares for $\mathrm{H} \alpha$.

results:

(i) The Gaussian decomposition fits indicate the existence of a central broad component of redshift consistent with the systemic velocity.

(ii) The presence of red- and blue-shifted broad components in the case of the considered lines implies that a part of the emission originates in a different region. This may indicate that part of the broad Balmer emission comes from a disc.

Some information about the kinematics of the BLR can be extracted from the Gaussian analysis even if it is very approximate. If the red- and blue-shifted Gaussians represent the two peaks of a disc-line profile, we can use the empirical relation given by Smak (1981) (see also Papkalla 1995):

$\Delta z=\frac{\Delta \lambda_{\mathrm{p}}}{\lambda_{0}} \approx \frac{V\left(R_{\text {out }}\right)}{c} \times \sin i$,

where $\Delta \lambda_{\mathrm{p}}$ is the difference between the two peaks, $\lambda_{0}$ the transition rest wavelength, $c$ the speed of light, and $V\left(R_{\text {out }}\right)$ the velocity at the outer radius of the disc that can be estimated by

$V\left(R_{\text {out }}\right) \approx \sqrt{\frac{2 G M}{R_{\text {out }}}}$.

Using Eqs. (1) and (2) we can obtain a very useful relation

$\sin i \approx \Delta z \sqrt{R_{\text {out }}^{\prime}}$,

where $R_{\text {out }}^{\prime}$ is given in Schwarzschild radii. From Eq. (3) we can estimate the maximal outer radius, since $\sin i \leq 1$. From Table $1,<\Delta z>>_{\mathrm{av}}=\left\{\left|z_{\mathrm{B}}\right|+\left|z_{\mathrm{R}}\right|\right\}_{\mathrm{av}}=0.01216$ and we obtain that $R_{\text {out }}^{\max }<7 \times 10^{3} R_{\mathrm{Sch}}$. On the other side, from previous investigations (e.g. Wandel et al. 1999; Popović et al. 2001) the outer radius of a BLR has typical dimensions of $\sim 1000 R_{\mathrm{Sch}}$, then we can estimate that $i \approx 23^{\circ}$.

\subsection{Two-component model for NGC 3516 Balmer Broad Emission Lines}

According to the results obtained from the Gaussian analysis as well as from previous works (Sulentic et al. 1998;
Nandra et al. 1999; Pariev 2001), in this section we will fit the Balmer lines using a two-component model based on a disc and a central Gaussian component which could come from a region surrounding the disk. For the disk we are going to use the Keplerian relativistic model of Chen \& Halpern (1989). The emissivity of the disk as a function of radius, $R$, is given by $\epsilon=\epsilon_{0} R^{-\mathrm{p}}$. We divide the disc into $n$ parts, assuming that all of them emit a Gaussian with the same width and shift that could be produced by an electron scattering atmosphere or by local turbulence (Chen \& Halpern 1989, see also Corbett et al. 1998).

Before performing the fit we have "cleaned" the $\mathrm{H} \alpha$ spectra by subtracting the narrow $\mathrm{H} \alpha+[\mathrm{NII}]$ lines and the $\mathrm{H} \beta$ spectra by subtracting the narrow [OIII] lines and the Fe II template.

The results of the fit are presented in Figs. 7-12 and the obtained disk and Gaussian parameters in Table 2. In all the cases we adopted an inclination $i \approx 11^{\circ}$ and a value for the emissivity parameter $p=3.15$ which fit the line profiles best.

We should mention here that changing the other parameters (inner, outer radius of the disc, emissivity parameter $p$ ) we can obtain a satisfactory fit with the inclination $11 \pm 5$ degrees.

From Table 2 and Fig. 12 we can point out the following results:

(i) There is a very good consistence for the different Balmer lines in the parameters $\left(<z>=-60 \mathrm{~km} \mathrm{~s}^{-1}\right.$ and $\langle W\rangle=$ $1480 \mathrm{~km} \mathrm{~s}^{-1}$, see Table 2, Cols. 6 and 7) of the broad component representing the region surrounding the disc. Its mean redshift exhibits a very small difference with respect to the cosmological redshift.

(ii) There is also a good consistence in the strong blueshift of the Gaussian from disc $\left(-760 \mathrm{~km} \mathrm{~s}^{-1}\right)$ with respect to the systemic velocity.

(iii) While the scatter in inner radius among the different Balmer lines seems not to be relevant, the ordered scaling in outer radius from $\mathrm{H} \epsilon$ to $\mathrm{H} \alpha$ could be significant.

Taking into account the estimated mass of the central object in NGC $3516\left(M \sim 4 \times 10^{7} M_{\odot}\right)$ given by Padovani et al. (1990) we can obtain the dimensions of the radiating disk: $R_{\text {inn }} \approx 0.004 \mathrm{pc}, R_{\text {out }} \approx 0.018 \mathrm{pc}$. The size of the whole BLR (disk + surrounding region) cannot be inferred from this analysis but it might be considerably larger. On the other hand, the BLR surrounding the disc may be originated from the accretion disc wind, that may be created due to several disturbances capable of producing shocks (e.g. a Bondi-Hoyl flow, stellar wind-wind collisions, and turbulences, see Fromerth \& Melia 2001). Recently, Fromerth \& Melia (2001) describe a scenario of the formation of BLR clouds in the accretion shocks, which can create a surrounding BLR.

We found a good agreement, for each considered line, between the disc model blue peak and the blueshifted component of the Gaussian decomposition (Fig. 12). This agreement is even better for the central Gaussian adopted in both models, while the redshifted component is blueshifted with respect to the disc model red peak. Besides this fact we do not insist in any detailed comparison of the disc-model and the Gaussians in the multi-Gaussian approach. 
Table 2. The parameters of Gaussian in disc and the Gaussian components which represent a surrounding region. $z_{\mathrm{D}}$ and $W_{\mathrm{D}}$ are the shift and width of a Gaussian from the disc, respectively (see text, Sect. 3.3.); $z_{\mathrm{G}}$ and $W_{\mathrm{G}}$ are the shift and width of the Gaussian which represents the emission of the surrounding region, respectively; $z_{\mathrm{NLR}}$ and $W_{\mathrm{NLR}}$ are parameters of the Gaussian which represents emission of the NLR. $W$ and $z$ are given in $\mathrm{km} \mathrm{s}^{-1} . R_{\mathrm{in}}, R_{\mathrm{out}}$ are the inner and outer radius of the emitting disc expressed in $R_{\mathrm{g}}=G M / c^{2}$, respectively.

\begin{tabular}{ccccccccc}
\hline \hline Line & $z_{\mathrm{D}}$ & $W_{D}$ & $R_{\text {inn }}\left(R_{\mathrm{g}}\right)$ & $R_{\text {out }}\left(R_{\mathrm{g}}\right)$ & $z_{\mathrm{G}}$ & $W_{\mathrm{G}}$ & $z_{\mathrm{NLR}}$ & $W_{N L R}$ \\
\hline $\mathrm{H} \alpha$ & -600 & 620 & 440 & 1550 & +50 & 1500 & 0 & 360 \\
$\mathrm{H} \beta$ & -860 & 770 & 340 & 1250 & -30 & 1630 & -90 & 390 \\
$\mathrm{H} \gamma$ & -880 & 885 & 350 & 1100 & +200 & 1520 & -70 & 410 \\
$\mathrm{H} \delta$ & -800 & 570 & 420 & 900 & -150 & 1530 & -73 & 360 \\
$\mathrm{H} \epsilon$ & -680 & 755 & 590 & 850 & -100 & 1200 & -90 & 300 \\
\hline$<B a l>$ av & -764 & 720 & 428 & 1130 & -6 & 1476 & -65 & 364 \\
$\mathrm{rms}$ & \pm 120 & \pm 126 & \pm 100 & \pm 284 & \pm 138 & \pm 162 & \pm 37 & \pm 42 \\
\hline
\end{tabular}

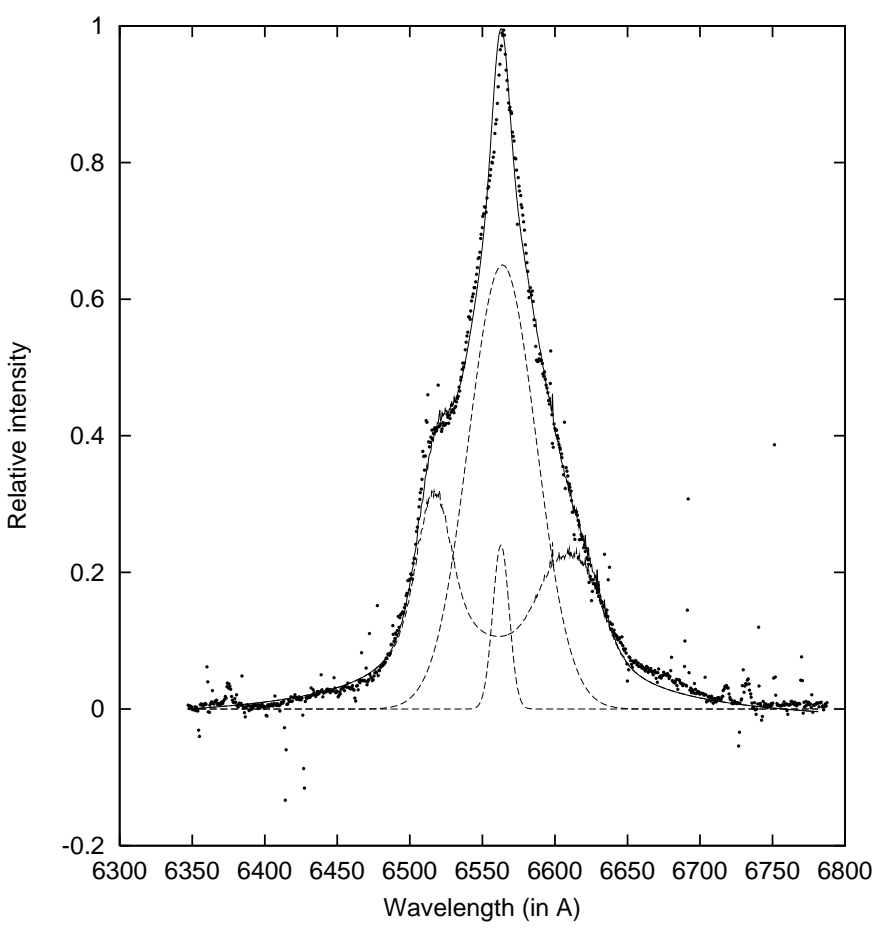

Fig. 7. The $\mathrm{H}_{\alpha}$ line fitted with the two-component model. The dots represent the observed and the solid line the best fit. The dashed broad lines represent the disc component, a broad component originates in an out-of-disc BLR and the narrow component from NLR, respectively.

\subsection{Physical properties in the NGC 3516 Balmer Line Emitting Region}

The plasma in the Balmer Broad Line Region probably does not come close to being in complete Local Thermodinamical Equilibrium (LTE), however, there may still be Partial Thermodinamical Equilibrium (PLTE) in the sense that populations of sufficiently highly excited levels are related to the next ion's ground state population by Saha-Boltzmann relations, or to the total population in all fine-structure levels of the ground-state configuration (see Griem 1968, 1997). For hydrogen lines various criteria were advanced for the minimum principal quantum number $n_{\mathrm{c}}$ for the lowest level, often called the thermal or collisional limit, for which PLTE remains valid. To conclude about presence of PLTE for Balmer lines, here we

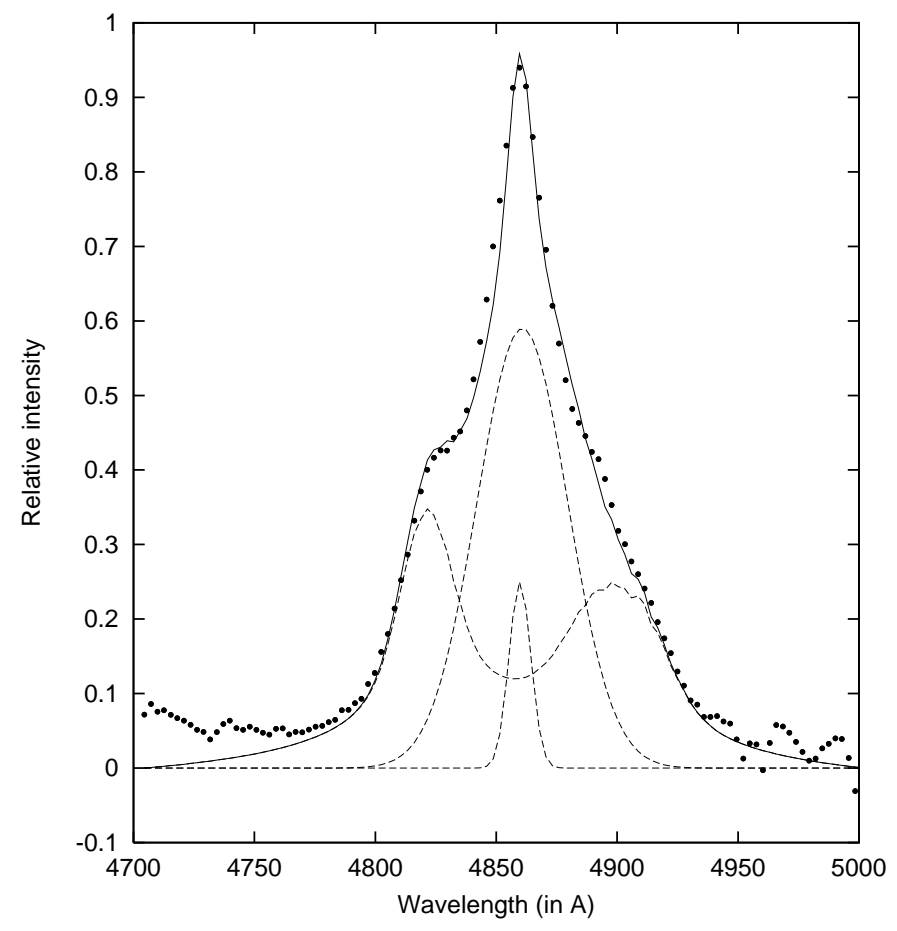

Fig. 8. The same as in Fig. 7, but for $\mathrm{H}_{\beta}$ line.

are going to use the method, well known in laboratory plasma diagnostic as Boltzmann plot method (see e.g. Griem 1997; Konjević 1999).

Taking into account that the Balmer lines originate from the same multiplet, as well as they have the same lower level, we can use the Boltzmann plot relation:

$\log \left(I_{n}\right)=\log \frac{I_{m n} \times \lambda_{m n}}{g_{n} A_{m n}}=B-A E_{n}$

where $I_{m n}$ is relative intensity of transition $n \rightarrow m, \lambda_{m n}$ is transition wavelength, $g_{n}$ is statistical weight of upper level, $A_{m n}$ is transition probability, $B$ and $A$ are constants, and $E_{n}$ is the energy of the upper level.

If we can approximate the $\log \left(I_{n}\right)$ as linear function of $E_{n}$ then

1) we can conclude that PLTE is at least some extent present in the Balmer Broad Line Emitting Region. 


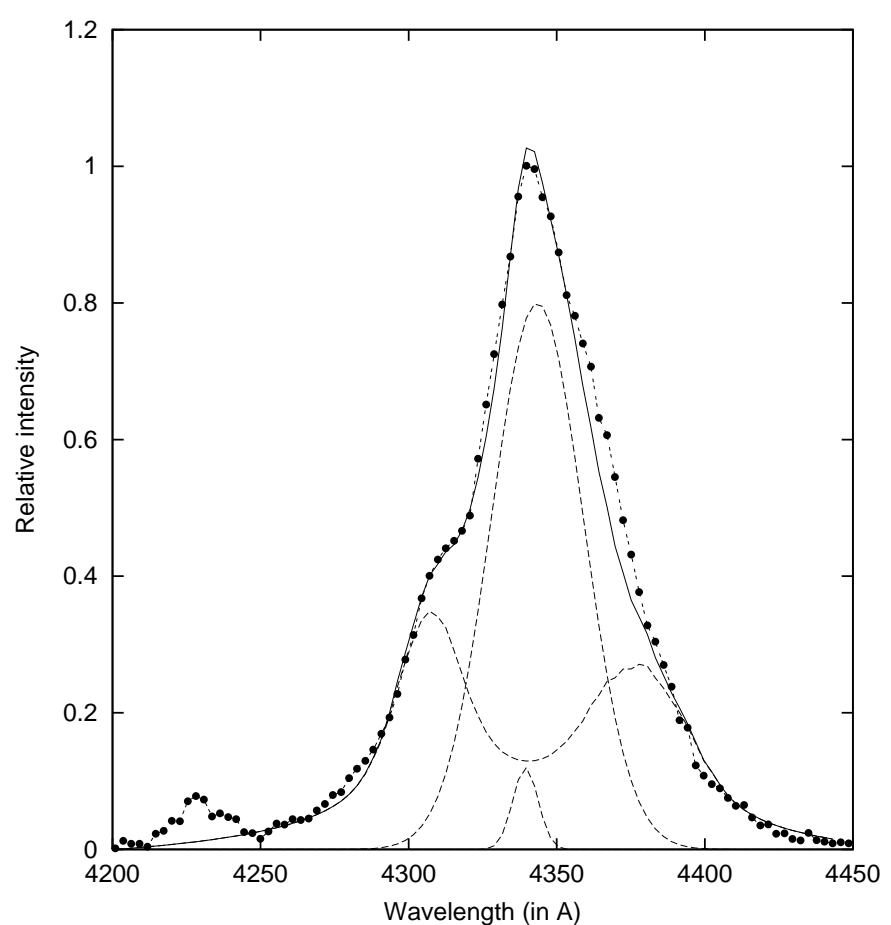

Fig. 9. The same as in Fig. 7, but for $\mathrm{H}_{\gamma}$ line.

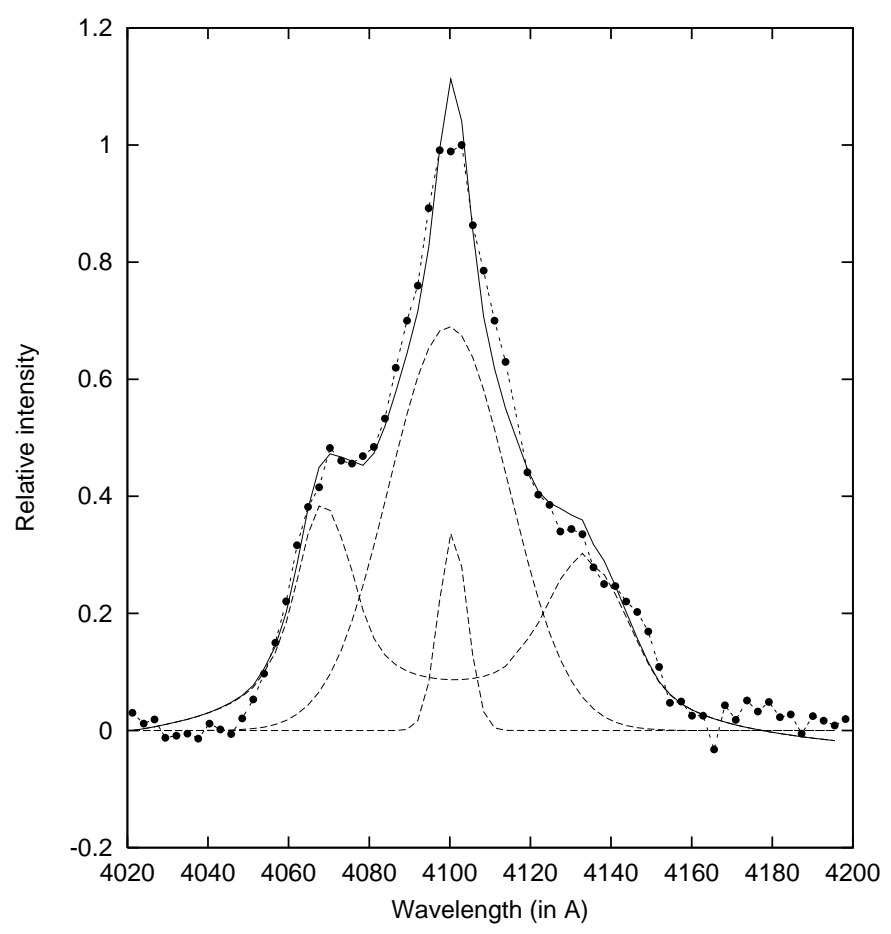

Fig. 10. The same as in Fig. 7, but for $\mathrm{H}_{\delta}$ line.

2) if PLTE is present the population adhering to a Boltzmann distribution is uniquely characterized by their excitation temperature. Then we can estimate the electron temperature from Eq. (4), $T=1 /(k A)$, where $k$ is the Boltzmann constant.

3) if PLTE is present we can estimate the electron density in Balmer broad line emitting region.

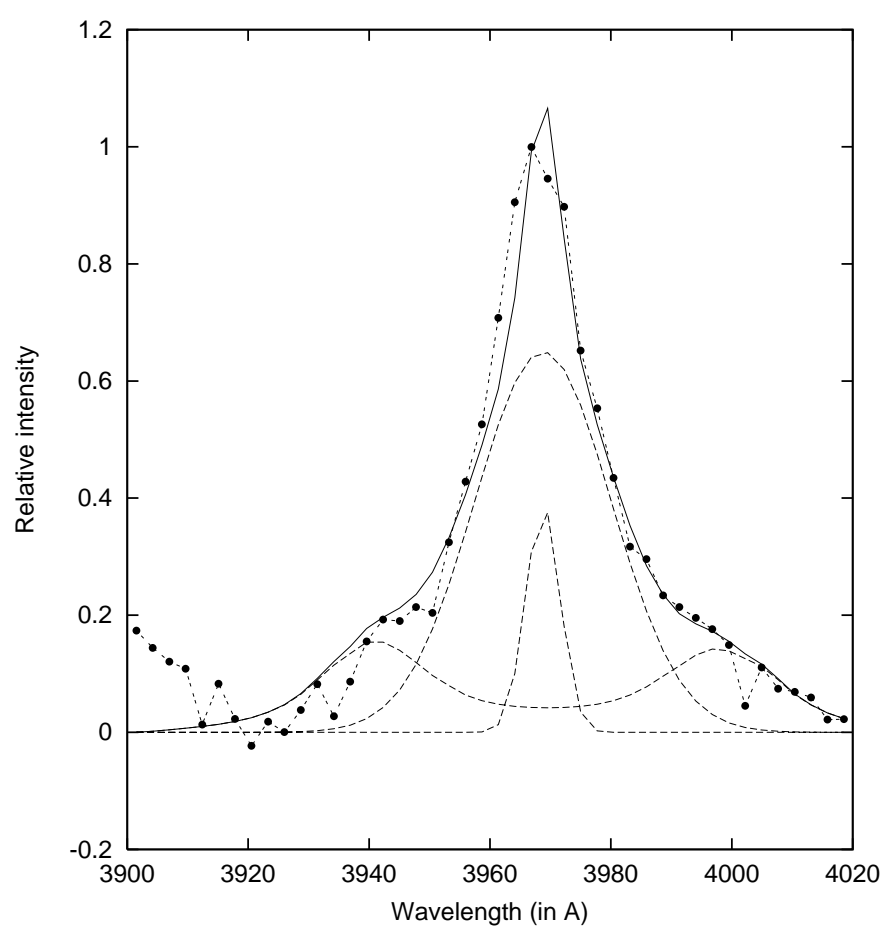

Fig. 11. The same as in Fig. 7, but for $\mathrm{H}_{\varepsilon}$ line.

In Fig. 13 we present our measurements. First we measured the total flux for each line (Fig. 13a), after that we measured the line flux which originated in the disc (Fig. 13c when central narrow and broad Gaussians were subtracted, Fig. 12). In Fig. $13 \mathrm{~b}$ the measurements of the surrounding region are presented. As one can see from Figs. 13a-c all three species indicate the the presence of PLTE (straight line as a function). From the fitting of the data with straight lines we estimated the electron temperature in these two separate regions. We found that the electron temperature in the disc was about $12500 \mathrm{~K}$, and in the surrounding region was around $20000 \mathrm{~K}$.

In a favorable situation the electron temperature may be determined by this method to $2-3 \%$, while normally an uncertainty of about 5-10\% must be expected for laboratory plasma (Konjević 1999). The determination of the temperatures is very sensitive to flux measurements. As one can see from Figs. 13a-c uncertainties in the measured flux are higher for weaker lines. Especially in the lines of the disc component where the flux is lower than the flux of the surrunding component. Consequently, the uncertainties in the temperature determination in this case are from $10 \%$ to $20 \%$. Valid criteria for PLTE and criteria for the application of this spectroscopic method is discussed by Griem (1997). For spatially homogeneous and inhomogeneous plasma, as well as in steady state plasma (applicable to slow time-varying plasma) it may be used to estimate the electron density (Griem 1997):

$N_{\mathrm{e}} \geq 7 \times 10^{18} Z^{6} n_{\mathrm{c}}^{-17 / 2} \frac{k T_{\mathrm{e}}}{E_{\mathrm{H}}} \mathrm{cm}^{-3}$

where $Z$ is the charge "seen" by optical electron $(Z=1$ for neutral emitters), $n_{\mathrm{c}}$ is the principal quantum number of the lower level and $E_{\mathrm{H}}$ is the ionization energy of Hydrogen. From this we estimated that electron density in both regions is of the 


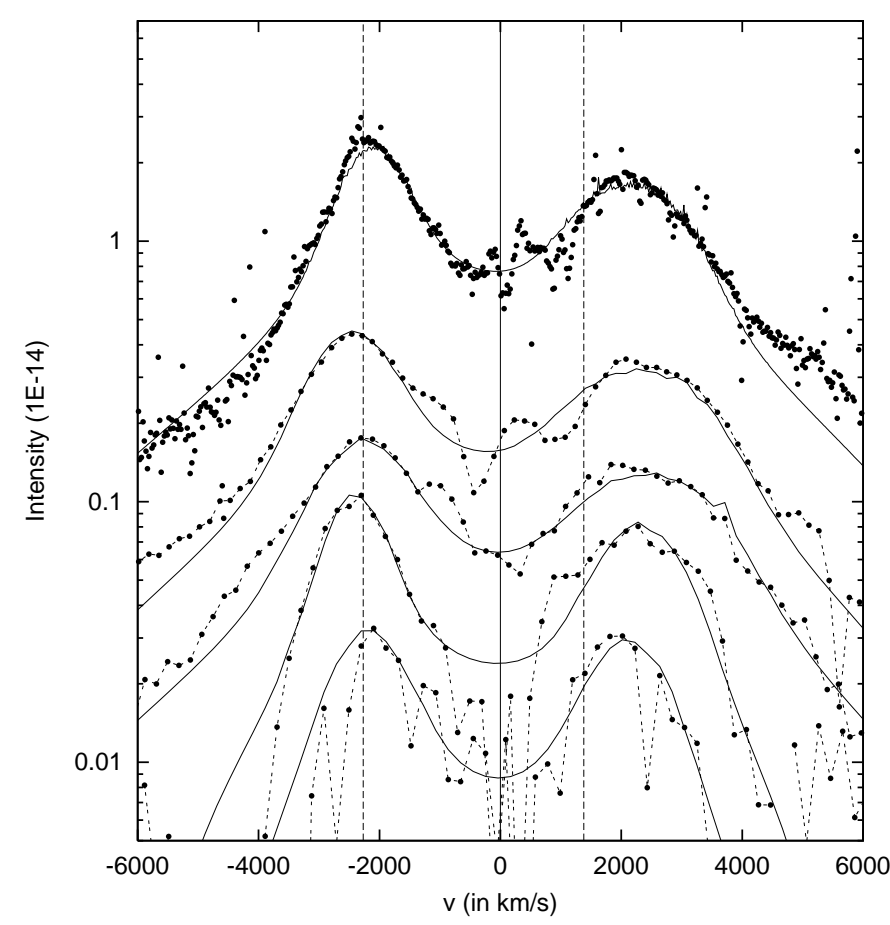

Fig. 12. The shapes of the Balmer lines, when the narrow and broad central components were subtracted (dots with dashed line) compared with the disc model obtained by fitting procedure (solid line). From bottom to top, $\mathrm{H} \varepsilon, \mathrm{H} \delta, \mathrm{H} \gamma, \mathrm{H} \beta, \mathrm{H} \alpha$. The dashed vertical lines indicate the averaged peak obtained from red- and blue-shifted Gaussian. The solid vertical line represents the cosmological redshift.

order of $10^{15} \mathrm{~cm}^{-3}$. Regarding the uncertainties of temperature the electron density is estimated to have an error of $10 \%$ to $20 \%$ too.

\section{Discussion}

From our analysis of the NGC 3516 Balmer lines we found that the shapes of these lines can be described by a two-component model including emission from a disc (or disc-like region) and a surrounding BLR. The parameters of the disc are in relatively good agreement with the previous results obtained for the $\mathrm{Fe} \mathrm{K}_{\alpha}$ (Nandra et al. 1997, 1999) and $\mathrm{H} \alpha$ line (Sulentic et al. 1998). We obtained a inclination for the disc of about $11^{\circ} \pm 5^{\circ}$ which is comparable with the results of Nandra (1999) $i \approx 0^{\circ}+19^{\circ}$ and Sulentic (1998) $i \approx 20^{\circ}$. However, it is in disagreement with the results obtained by Pariev et al. (2001) who found $i \approx 27^{\circ} \pm 4^{\circ}$, but taking into account the effect of e.g. warping (Bachev 1999; Hartnoll \& Blackmen 2000) the inclination of the Balmer Emission Line disc is in principle in good agreement with one obtained from the $\mathrm{Fe} \mathrm{K} \alpha$ line. Regarding the dimensions of the accretion region, we infer a size for the Balmer Line disc $\left(\sim 1100 R_{\mathrm{g}}\right)$ larger than that of the $\mathrm{Fe} \mathrm{K}_{\alpha}$ disc, but two orders of magnitude smaller than that estimated by Sulentic et al. (1998) for $\mathrm{H} \alpha, R_{\text {out }} \approx 1.5 \times 10^{5} R_{\mathrm{g}}$.

On the other hand, using the fact that the Balmer lines are emitted from the same multiplet, we found that PLTE exists, and we estimate the electron temperature of the disc (or disc-like) region and of the surrounding Balmer BLR to be around $12500 \mathrm{~K}$ and $20000 \mathrm{~K}$, respectively. This results are
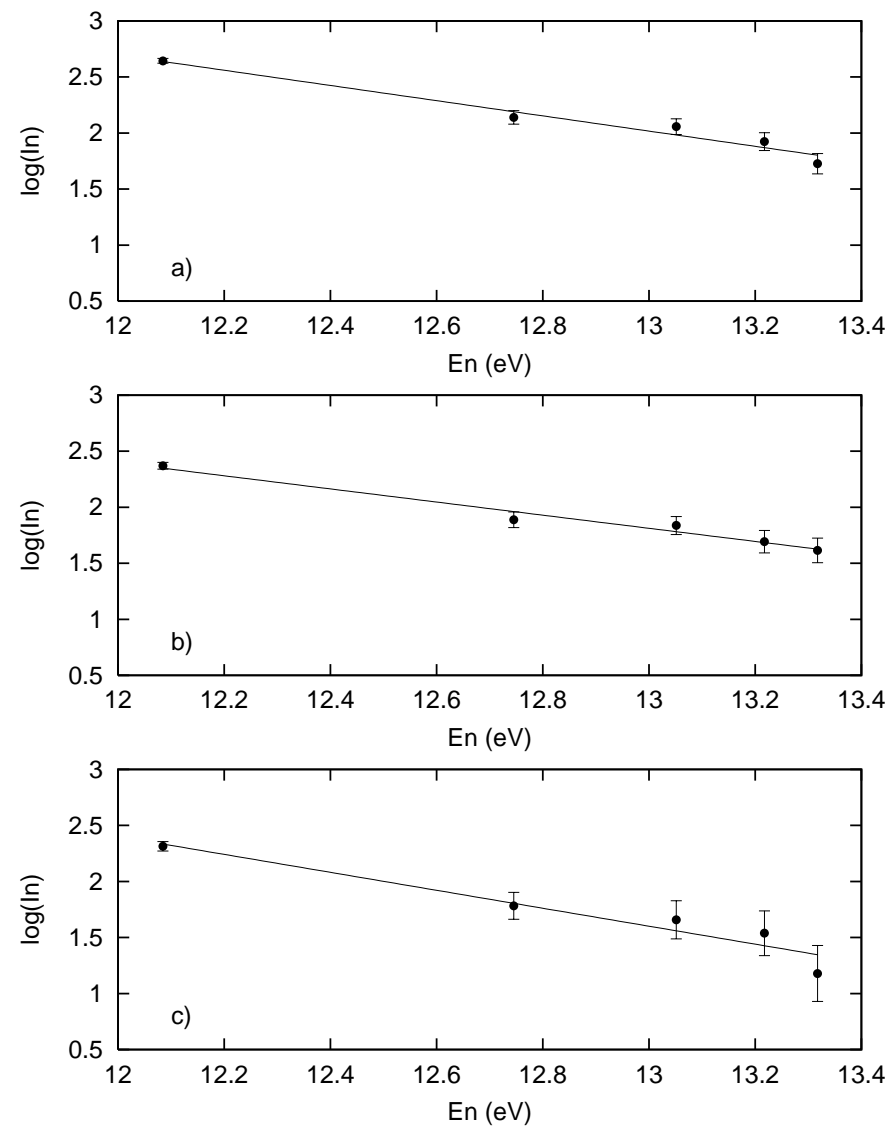

Fig. 13. The Boltzmann plot for the Balmer lines: a) total flux of the lines; $\mathbf{b}$ ) the flux of the surrounding region and $\mathbf{c}$ ) the flux of the emission disc.

in agreement with previous estimations (see, e.g. Osterbrock 1993; Sulentic et al. 2000). The electron density estimated by us, $\sim 10^{15} \mathrm{~cm}^{-3}$, is significantly higher than in some previous estimations for the BLR (see, e.g. Osterbrock 1993; Sulentic et al. 2000). However, in other earlier works a high electron density have been also suggested (van Groningen 1987; Sivron \& Tsuruta 1993; Brotherton et al. 1994).

\section{Conclusions}

We have analyzed the Balmer lines to investigate the kinematical and physical properties of the Balmer Broad Line Region in NGC 3516. We have studied the application of a twocomponent model (disc, or disc-like region + a surrounding emission region) to the line profiles, finding:

1) The agreement among the parameters for the disc $(i \sim$ $11^{\circ} \pm 5^{\circ}, R_{\mathrm{inn}} \approx 400 R_{\mathrm{g}}$ ) and for the surrounding component (random velocity around $1000 \mathrm{~km} \mathrm{~s}^{-1}$ ) obtained from the fits to all the Balmer lines consistently support the hypothesis that a part of the BLR emission comes from a Keplerian disc.

2) The outer radius increases monotonically from $\mathrm{H} \epsilon$ to $\mathrm{H} \alpha$, showing possible evidences of the disc radial stratification.

3) Using the fact that the Balmer lines belong to the same multiplet and that they have the same lower level in the transition, and applying the Boltzmann plot method to estimate the electron temperature and density of the emitting disc and of the 
corresponding surrounding region, we found that the electron temperature in the disk is about $12500 \mathrm{~K}$ and in the surrounding region is around $20000 \mathrm{~K}$

4) We estimated that the electron density in both regions is about $10^{15} \mathrm{~cm}^{-3}$.

Acknowledgements. This work was supported by the Ministry of Science, Technologies and Development of Serbia through the project "Astrophysical Spectroscopy of Extragalactic Objects". We thank to Prof. S. Djeniže for a useful discussion regarding Boltzman-plot method.

\section{References}

Brotherton, M. S., Wills, B. J., Steidel, R., \& Sargent, W. L. W. 1994, ApJ, 423, 131

Bachev, R. 1999, A\&A, 348, 71

Chen, K., Halpern, J. P. \& Filippenko, A. V. 1989, ApJ, 339, 742

Chen, K., \& Halpern, J. P. 1989, ApJ, 344, 115

Chiang, J., \& Blaes, O. 2001, ApJ, 557, L15

Corbett, E. A., Robinson, A., Axon, D. J., Young, S., \& Hough, J. H. 1998, MNRAS, 296, 721

Edelson, R., Koratkar, A., Nandra, K., et al. 2000, ApJ, 534, 180

Eracleous, M., \& Halpern, J. P. 1994, ApJSS, 90, 1

Eracleous, M., \& Halpern, J. P. 2001, ApJ, 554, 240

Eracleous, M. 1999, in Structure and Kinematics of Quasar Broad Line Regions, ed. C. M. Gaskell, W. N. Brandt, M. Dietrich, D. Dultzin-Hacyan, \& M. Eracleous ASP Conf. Ser., 175, 163

Fromerth, M. J., \& Melia, F. 2001, ApJ, 549, 205

Goad, M. R., Koratkar, A. P., Axon, D. J., Korista, K. T., \& O’Brien, P. T. 1999, ApJ, 512 L95

Golev, V., Yankulova, I., Bonev, T., \& Jockers, K. 1995, MNRAS, 273, 129

Griem, H. R. 1968, Plasma Spectroscopy (McGraw-Hill, New York)

Griem, H. R. 1997, Principles of Plasma Spectroscopy (Cambridge Univ. Press, Cemridge)

van Groningen, E. 1987, A\&A, 186, 103
Hartnoll, S. A., \& Blackman, E. G. 2000, MNRAS, 317, 880

Konjević, N. 1999, Phys. Rep., 316, 339

Korista, K. T. 1992, ApJSS, 79, 285

Nandra, K., George, I. M., Mushotzky, R. F., Turner, T. J., \& Yaqoob, T. 1997, ApJ, 477, 602

Nandra, K., George, I. M., Mushotzky, R. F., Turner, T. J., \& Yaqoob, T. 1999, ApJ, 523, L17

Osterbrock, D. E. 1993, ApJ, 404, 551

Padovani, P., Burg, R., \& Edelson, R. A. 1990, ApJ, 353, 438

Papkalla, R. 1995, A\&A, 295, 551

Pariev, V. I., Bromley, B. C., \& Miller, W. A. 2001, ApJ, 547, 649

Pérez, E., Mediavilla, E., Penston, M. V., et al. 1988, MNRAS, 230, 353

Popović, L. Č., \& Mediavilla, E. G. 1997, Publ. Astron. Obs. Belgrade, 57, 95

Popović, L. Č., Stanić, N., Kubičela, A., \& Bon, E. 2001, A\&A, 367, 780

Popović, L. Č., Mediavilla, E., Bon, E., Stanić, N., \& Kubičela, A. 2002, A\&A, submitted

Reeves, J. N., Turner, M. J. L., Pounds, K. A., et al. 2001, A\&A , 365, L134

Sivron, R., \& Tsuruta, S. 1993, ApJ, 402, 420

Smak, J. 1981, Acta Astron., 31, 395

Storchi-Bergmann, T., Eracleous, M., Ruiz, M. T., et al. 1997, ApJ, 489,87

Sulentic, J. W., Marziani, P., Zwitter, T., et al. 1998, ApJ, 501, 54

Sulentic, J. W. 1999, in Structure and Kinematics of Quasar Broad Line Regions, ed. C. M. Gaskell, W. N. Brandt, M. Dietrich, D. Dultzin-Hacyan, \& M. Eracleous, ASP Conf. Ser., 175, 175

Sulentic, J. W., Marziani, P., \& Dultzin-Hacyan, D. 2000, ARA\&A, 38,521

Véron-Cetty, M.-P., \& Véron, P. 2000, A Catalogue of Quasars and Active Galactic Nuclei, Sci. Rep., 19

Wandel, A., Peterson, B. M., \& Malkan, M. A. 1999, ApJ, 526, 579

Wiese, W. L., Smith, M. W., \& Glennon, B. M. 1966, Atomic Transition Probabilities: Hydrogen Through Neon (NSRDS, Washington DC), vol. I 